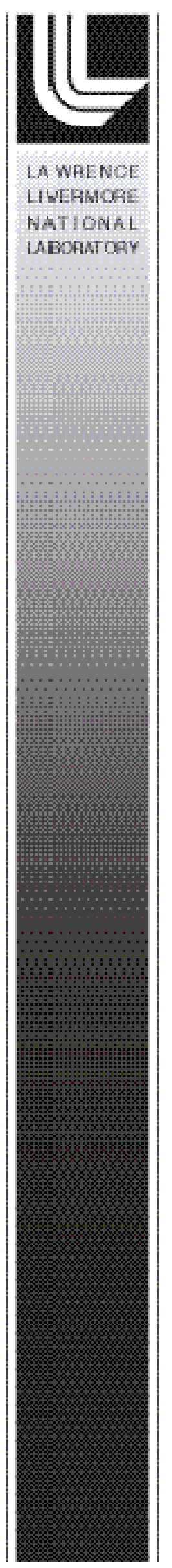

\title{
Methods for Calibration of Prout- Tompkins Kinetics Parameters Using EZM Iteration and GLO
}

\author{
A. P. Wemhoff \\ A. K. Burnham
}

November 8, 2006

This work was performed under the auspices of the U. S. Department of Energy by the University of California, Lawrence Livermore National Laboratory, under Contract No. W-7405-Eng-48. 


\section{DISCLAIMER}

This document was prepared as an account of work sponsored by an agency of the United States Government. Neither the United States Government nor the University of California nor any of their employees, makes any warranty, express or implied, or assumes any legal liability or responsibility for the accuracy, completeness, or usefulness of any information, apparatus, product, or process disclosed, or represents that its use would not infringe privately owned rights. Reference herein to any specific commercial product, process, or service by trade name, trademark, manufacturer, or otherwise, does not necessarily constitute or imply its endorsement, recommendation, or favoring by the United States Government or the University of California. The views and opinions of authors expressed herein do not necessarily state or reflect those of the United States Government or the University of California, and shall not be used for advertising or product endorsement purposes. 
UCRL-TR-225940

\section{Abstract}

This document contains information regarding the standard procedures used to calibrate chemical kinetics parameters for the extended Prout-Tompkins model to match experimental data. Two methods for calibration are mentioned: EZM calibration and GLO calibration. EZM calibration matches kinetics parameters to three data points, while GLO calibration slightly adjusts kinetic parameters to match multiple points.

Information is provided regarding the theoretical approach and application procedure for both of these calibration algorithms. It is recommended that for the calibration process, the user begin with EZM calibration to provide a good estimate, and then fine-tune the parameters using GLO. Two examples have been provided to guide the reader through a general calibrating process. 
UCRL-TR-225940

\section{Table of Contents}

About the Extended Prout-Tompkins Model................................................ 5

Background of the EZM and GLO Calibration Methods .................................. 7

Single-Parameter - Single Point Iterative Calibration ....................... 8

Coupled erx-zrx Two Point Iterative Calibration ................................ 13

EZM Three Point Iterative Calibration .............................................. 14

GLO Calibration ........................................................................... 16

Running the Calibration Codes .................................................................... 18

Performing a Point-by-Point EZM Calibration .................................. 23

Performing a GLO Calibration...................................................... 24

Running a Set of Sequential Runs with the Same Kinetic Parameters .... 25

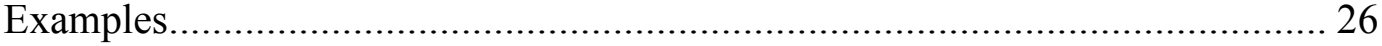

Example 1: Two-Point Calibration................................................. 26

Example 2: Three-Point Calibration ................................................ 29

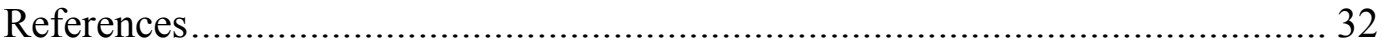


UCRL-TR-225940

\section{About the Extended Prout-Tompkins Model}

A simple, universal, and functionally accurate kinetics model of the thermal decomposition of various explosives would save computational resources for the incorporation of non-traditional explosives into large-scale cookoff simulations. Wemhoff and Burnham [1] have explored substituting a sequential reaction process by a single reaction model that incorporates aspects of nucleation and propagation: the extended Prout-Tompkins model [2],

$$
\frac{d x}{d t}=-k x^{n}(1-q x)^{n}=-k(1-q x)
$$

where $x$ is the fraction remaining of reactant, $t$ is time, and

$$
k(T)=A \exp \left(-\frac{E}{R T}\right)
$$

where $A$ is the frequency factor in units of reciprocal time and $E$ is the activation energy in units of energy per mole. In this document, for clarity we introduce the parameter $p$,

$$
p=-\log _{10}(1-q)
$$

which may also be solved for $q$ as

$$
q=1-10^{-p}
$$

which contains the property that if $p$ contains no nonzero values after the decimal point, then it is identical to the number of 9's after the decimal point for $q$. For example, for $q=$ $0.9999, p=4.0$.

In order to use the above kinetics model, Wemhoff and Burnham [1] explored the effects of adjusting the various parameters on the time to explosion for the ODTX experiment. Therefore, several test runs of RDX were performed to determine the effect of varying these parameters. In these runs, the parameters $E / R$ and $A$ were maintained at $20000 \mathrm{~K}$ and $1 \times 10^{15}$ per sec, respectively, except in a single case where $A$ was adjusted to match the highest temperature data point between curves for $n=0, m=1$ and $n=0, m=0$. The two extreme values of $p$ were used in Figures $1 \mathrm{a}$ and $1 \mathrm{~b}$ ( 2 and 9 , respectively), where any value of $q$ less than 2 is unphysical per the Prout-Tompkins approximation (see [1]), while any value higher than 9 is read as $q=1$ into ALE3D, which causes no reaction to proceed per Eq. (1). The values of $n$ used were 0.0 and 1.0, while the values of $m$ used were $0.0,0.5$ and 1.0. These curves suggest the following:

- Increasing the parameter $p$ tends to flatten out the ODTX trend and generally increases the time to explosion (i.e. it shifts the curve upwards). 
- Increasing the parameter $m$ also tends to flatten out the curve and shifts it upward in a manner similar to increasing $p$, although the value of $m$ has a more pronounced effect on the location of the bend in the curve.

- Increasing the parameter $n$ has very little effect on the calculation except near the critical temperature.

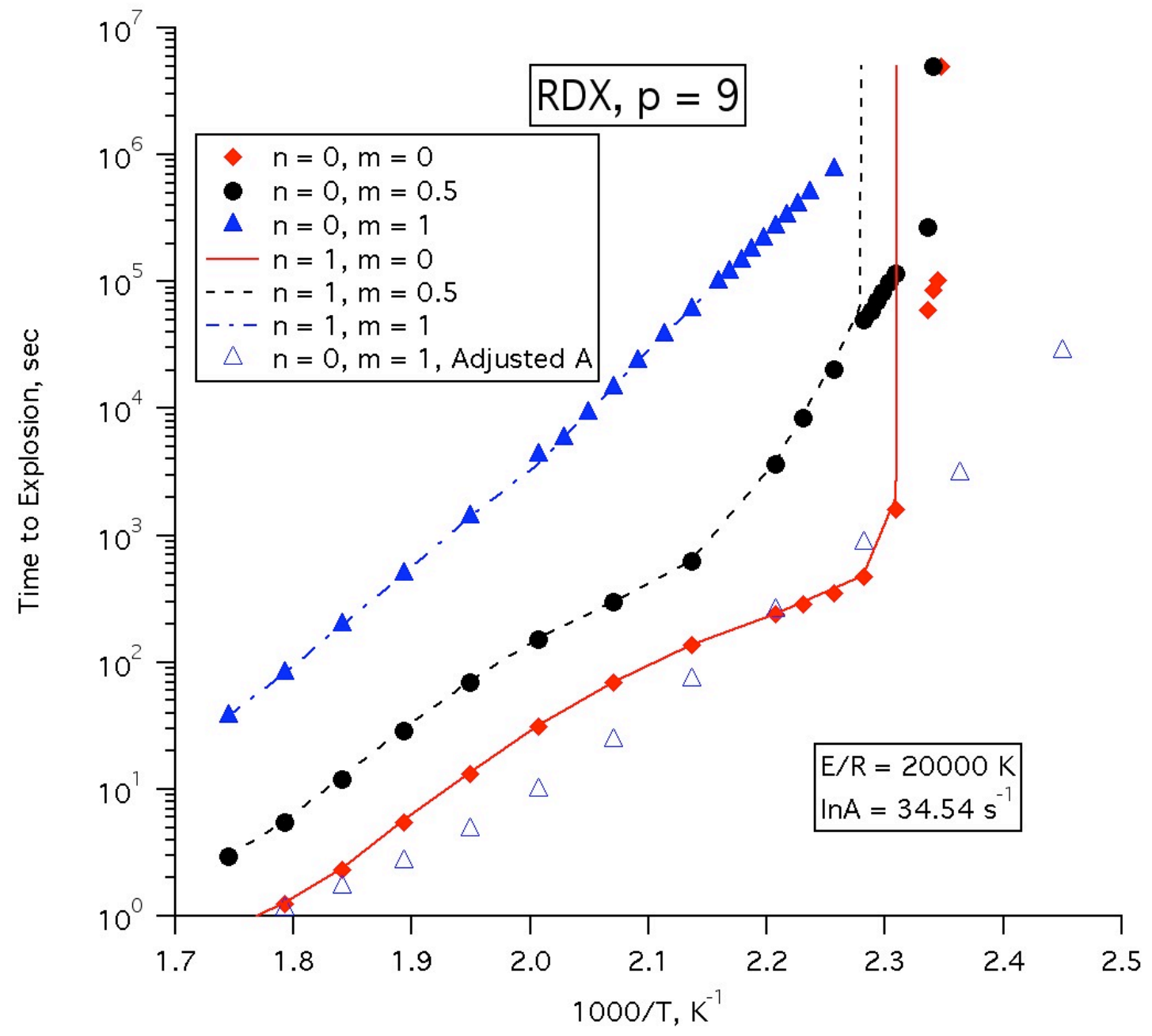

FIGURE 1a. Simulated ODTX trends for RDX for $p=9$. Value of adjusted frequency factor for open triangles is $1.04 \times 10^{18}$ per sec. 
UCRL-TR-225940

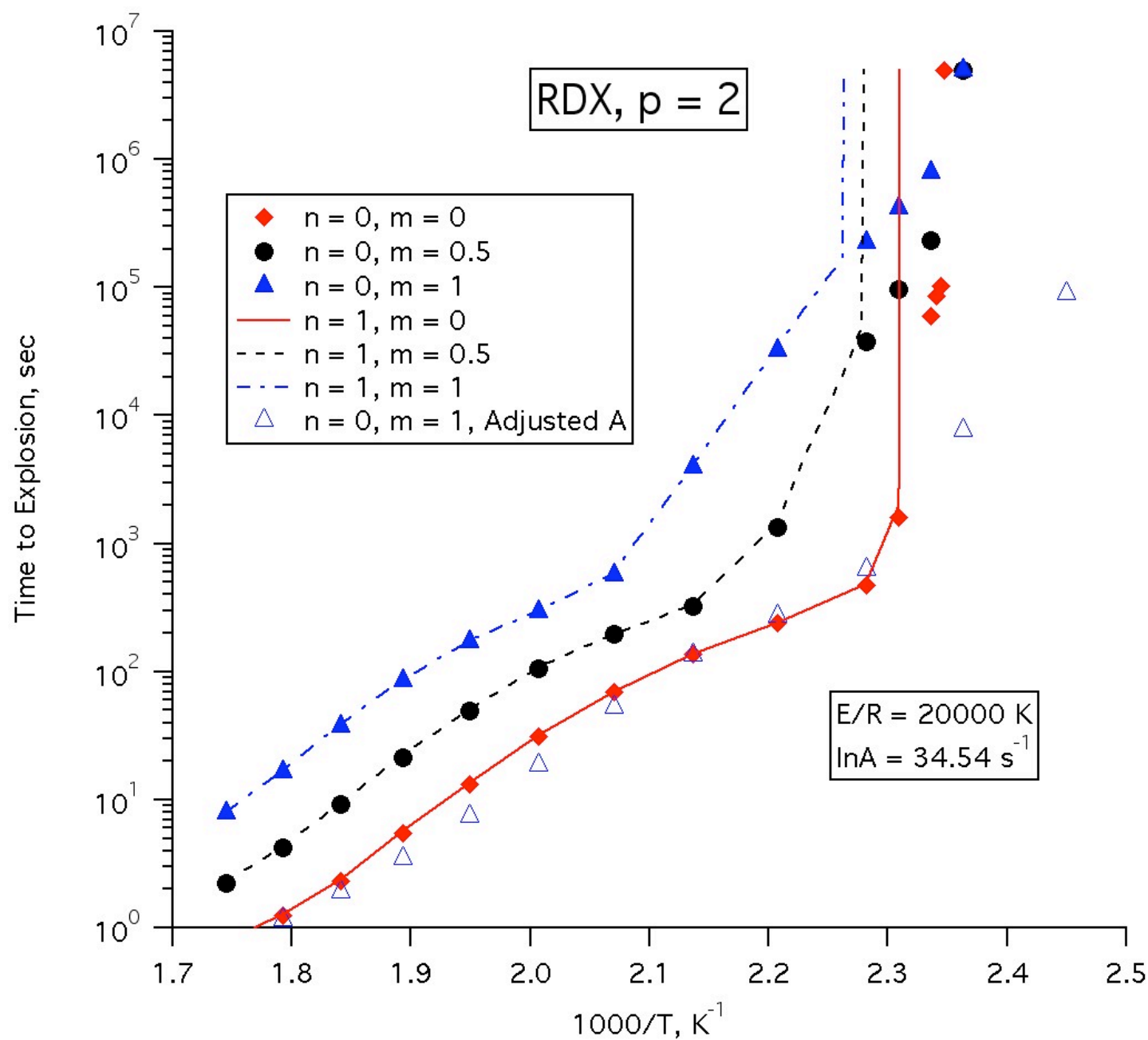

FIGURE 1b. Simulated ODTX trends for RDX for $p=2$. Value of adjusted frequency factor for open triangles is $5.70 \times 10^{16}$ per sec.

\section{Background of The EZM and GLO Calibration Methods}

The general knowledge of the characteristics of varying these parameters allows for the choice of calibration techniques for a given explosive. For example, Figure 1a shows that the curvature of the ODTX curve reduces as $p$ increases. In addition, Figure $1 \mathrm{~b}$ shows that the choice of $m$ has a large influence on the location of the bend in the ODTX curve. Therefore, knowledge of the calibration data allows for two general approaches for calibration:

- If the experimental data appear in an approximately straight line, then set the value of $p$ to its highest allowable value (generally 9) after the decimal point, $n=$ 0 , and $m=1$. $A$ and $E$ are the primary parameters optimized. The initial use of $n$ $=0$ is preferred for numerical stability. 
- If the experimental data contain a large increase in explosion times at low temperatures, then set the value of $p$ to a lower number (generally 3 ) and adjust the values of $A, E$, and $m$ accordingly to create the bend in the simulated curve.

The approach for each explosive generally falls under one of the two above strategies. The dependence on $n$ is sufficiently weak that it may be optimized in a final fine-tuning to match low-temperature data if necessary. However, ODTX data is usually insufficient to constrain $n$, and its value is probably best determined by independent evidence.

Thermal analysis data supports $n$ values closer to unity. Better guidance is a matter of current research.

The first step in calibration is to determine representative data points for use in the simulations. The LLNL Explosives Guide [3] provides experimental ODTX results for a wide variety of explosives and temperatures. Generally, one or several of these data points are used for a given calibration technique, and the choice of data points should provide a reasonable approximation of the explosion time-temperature curve.

There are several approaches for calibrating the parameters in Eq. (1) to match ODTX data. These calibration techniques are now described, ranging from simplest to most complex. The increase in complexity in the iterative calibration techniques is meant to follow an increase in usefulness of the techniques for attaining accurate parameters. In ALE3D, the Prout-Tompkins reaction is generally input as

$$
k\left(x_{s}, T\right)=\exp \left(z r x-\frac{e r x}{R T}\right) x^{n}(1-q x)^{m}
$$

where $e r x=E$ in cal $/ \mathrm{mol}$, and $z r x=\ln \left(10^{-6} A\left[\mathrm{~s}^{-1}\right]\right)$. It should be noted that the parameter rhopow $=1-m-n$ is used to convert the Prout-Tompkins reaction from the default mass concentration basis to a mass fraction basis in ALE3D. The only reaction considered in this study is the direct decomposition of solid material to gaseous products, and therefore melting was not taken into account. Therefore, the parameters to be calibrated are $z r x$, $e r x, m, n$, and $q$.

\section{Single Parameter - Single Point Iterative Calibration}

This algorithm allows the user to calibrate the parameters in ALE3D by adjusting a single kinetics parameter to match results for a single datum. In this simple program, the user inputs the following variables:

- parameter to be optimized

- goal time in seconds

- maximum number of iterations

- minimum and maximum parameter value

- run temperature

- convergence tolerance

- parameter adjustment algorithm 
The code first checks that the goal may be reached by determining explosion times for the minimum and maximum parameter values. If the goal lies between these calculated explosion times, then calibration is possible. An example is shown schematically in Figure 2, where the time-to-explosion $t$ increases as the parameter $m$ increases, and the time-to-explosion goal $t_{\text {goal }}$ is found to exist for a parameter value in the range $m_{\min }<m<$ $m_{\max }$, assuming that there exists only one value of $t$ for a given $m$. Note that the descriptors $\min$ and $\max$ refer to the parameter $m$ only; i.e. the value of $t_{\max }$ may be lower than $t_{\min }$ if the general slope of the curve is negative.

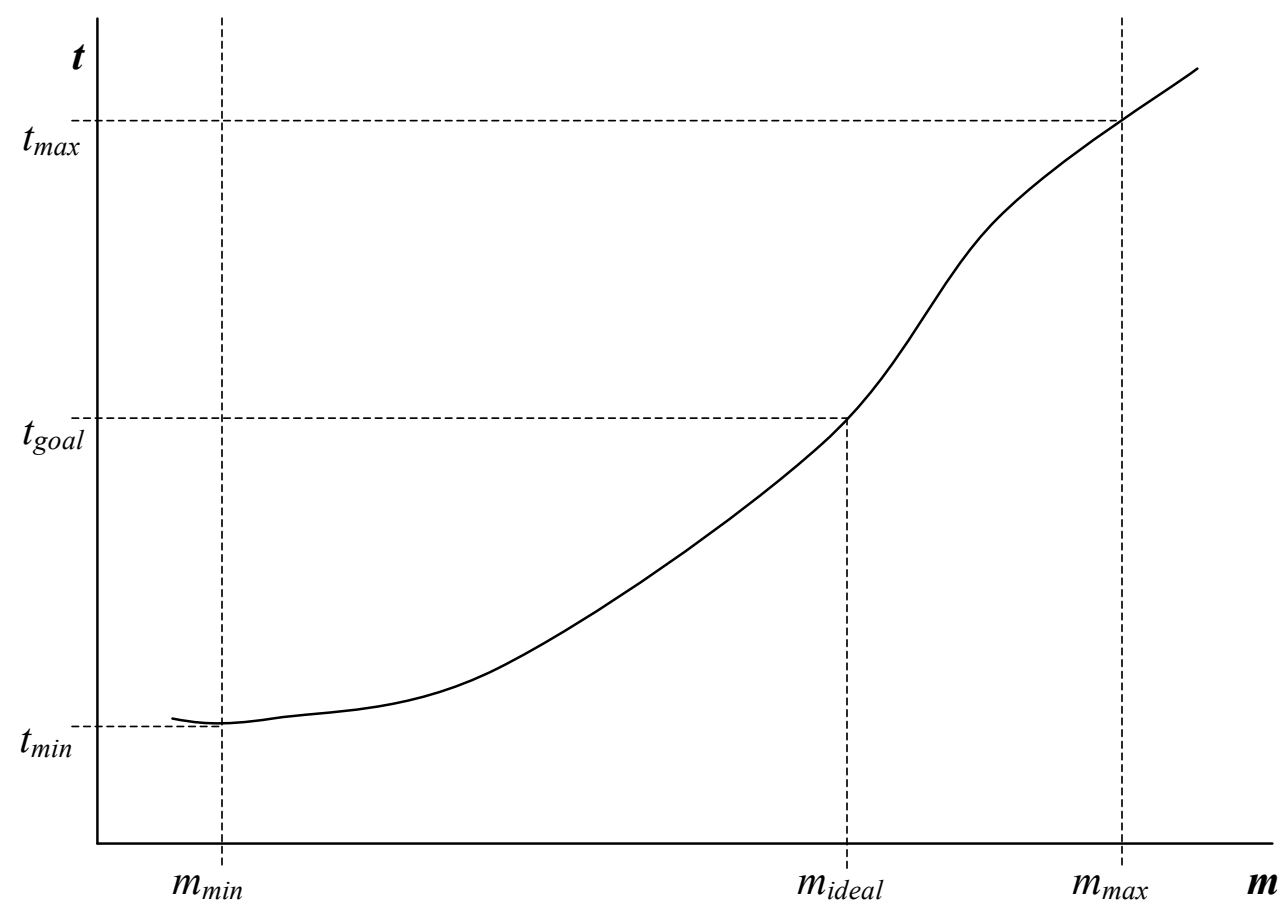

FIGURE 2. Schematic of example relationship between $m$ and $t$.

The code attempts to calibrate the parameter in two ways. These procedures are repeated until either the maximum number of iterations or the convergence criterion has been met. The linear interpolation algorithm is the default method for all iterative calibration techniques.

- Simple bracketing: if none or only one of the calculated times-to-explosion are known for the edges of the bracketing window, then the new parameter value is at the center of the bracketing window as shown in Figure 3, and mathematically by

$$
m_{\text {new }}=\frac{1}{2}\left(m_{\min }+m_{\max }\right)
$$


For each iteration, the algorithm also replaces the value of either $m_{\min }$ or $m_{\max }$ based upon the calculated value of $t$, which reduces the bracketing window by one-half during each iteration.

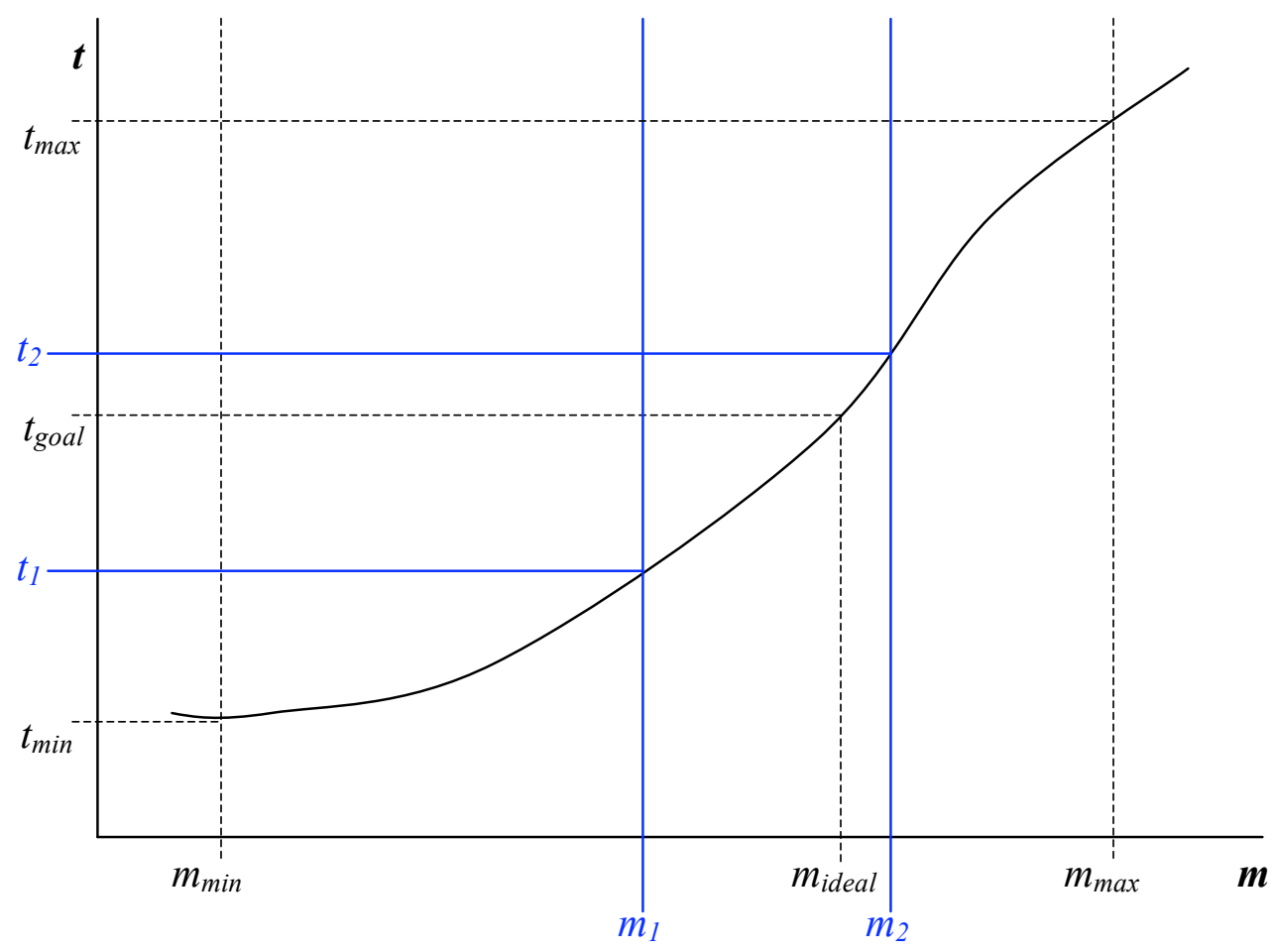

FIGURE 3. Simple bracketing algorithm schematic.

- Linear interpolation: if both calculated times-to-explosion are known at the edges of the bracketing window, then the new parameter value is found using linear interpolation as shown in Figure 4. The updated value is found by

$$
m_{\text {new }}=m_{\text {min }}+\left(m_{\text {max }}-m_{\text {min }}\right) \frac{\left(t_{\text {goal }}-t_{\text {min }}\right)}{\left(t_{\text {max }}-t_{\text {min }}\right)}
$$


UCRL-TR-225940

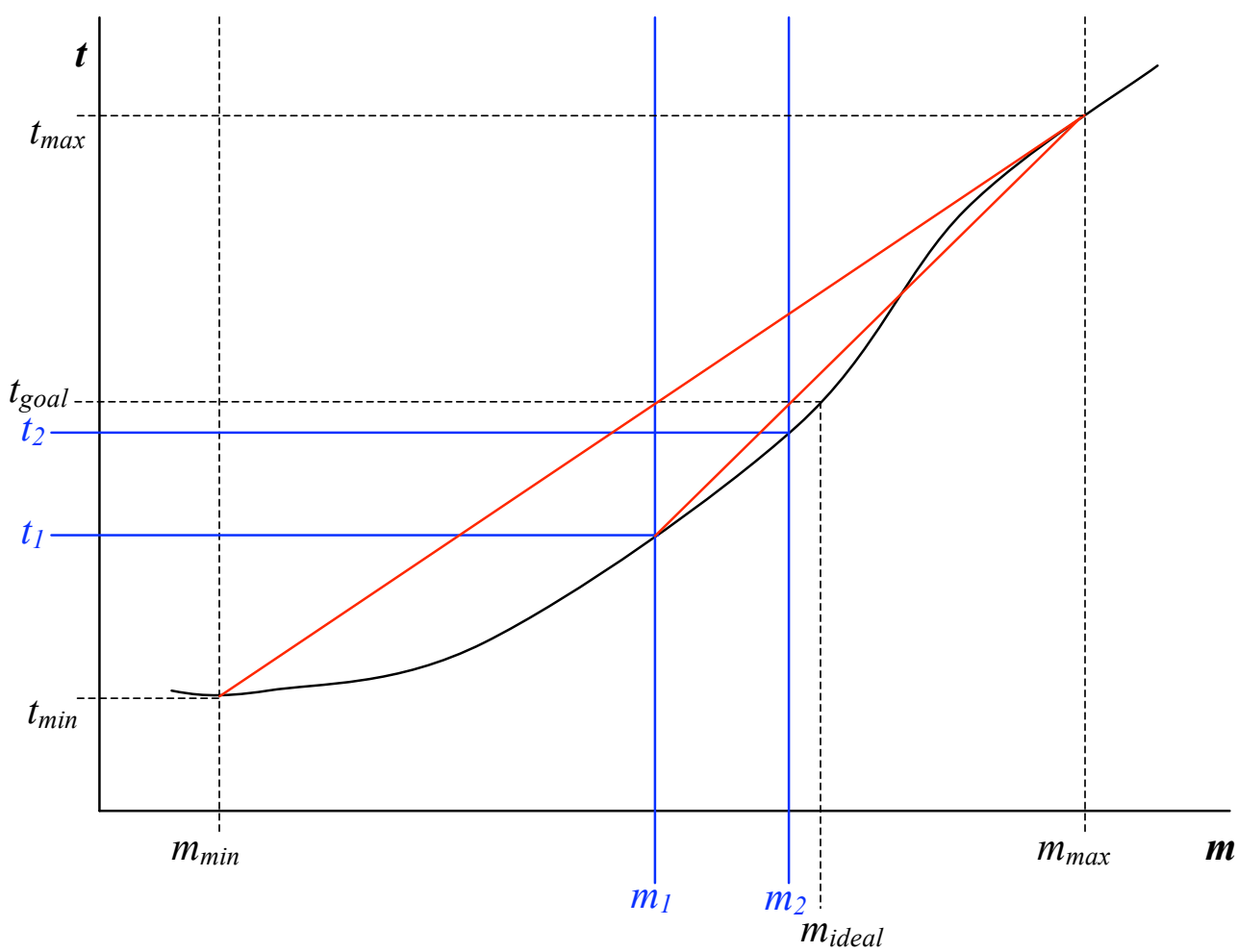

FIGURE 4. Linear interpolation method schematic.

- Shifted linear interpolation: this algorithm provides a modification to the standard linear interpolation algorithm to take curvature effects into account. An example of this is shown in Figure 5, where the large curvature of the time curve shifts the value of $m_{2}$ from $m_{2 L}$ to $m_{2 P}$. This algorithm is only implemented when the calculated time is far from the goal and when no shifting had taken place the previous time step. An applied relaxation factor continually updates after each shifting step to increase accuracy. Table 1 shows that studies of this algorithm have shown significant reduction in the required number of iterations compared to where no shifting was applied when the curvature of the dependent variable curve is large. 
UCRL-TR-225940

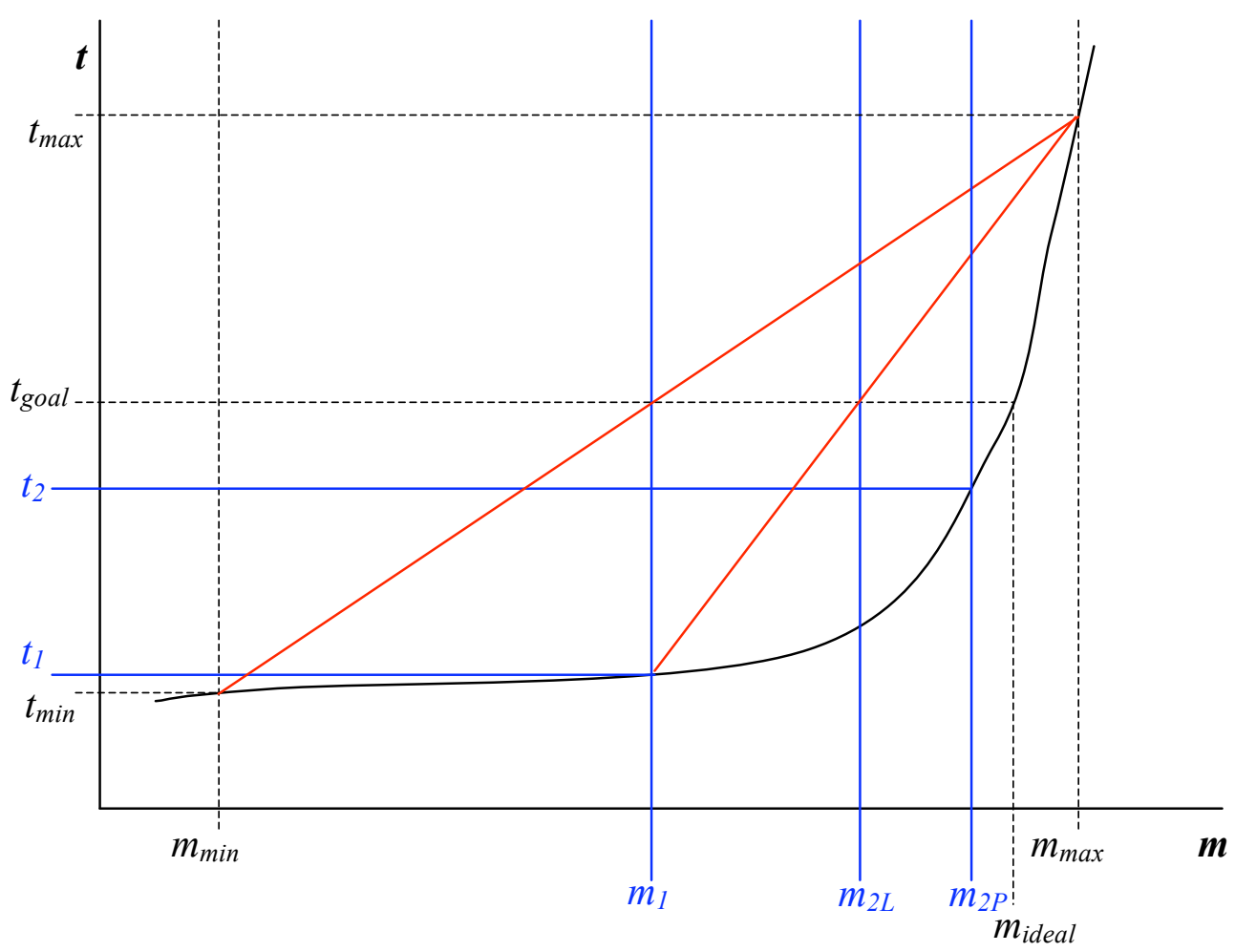

FIGURE 5. Shifted linear interpolation method schematic.

TABLE 1. Comparison of convergence efficiency from application of shifting algorithm. The range for all equations is $0.1<x<5.0$, the goal is 15.0, and the convergence tolerance is $1 \%$.

\begin{tabular}{|c|c|c|}
\hline \multirow{2}{*}{$\begin{array}{c}\text { Equation } \\
t=f(m)\end{array}$} & \multicolumn{2}{|c|}{ Steps Required for Convergence } \\
\hline & $\begin{array}{c}\text { Linear Interpolation } \\
\text { Without Shifting } \\
\end{array}$ & $\begin{array}{c}\text { Linear Interpolation with } \\
\text { Shifting }\end{array}$ \\
\hline$t=\exp (m)$ & 23 & 6 \\
\hline$t=m^{2}$ & 5 & 5 \\
\hline$t=m^{3}$ & 15 & 7 \\
\hline$t=1000 \exp (-m)$ & 67 & 8 \\
\hline$t=100 \ln (1+m)$ & 5 & 4 \\
\hline
\end{tabular}




\section{Coupled erx-zrx Two Point Iterative Calibration}

The single parameter-single point iterative calibration tool assumes no coupling between the parameters. However, the compensation relationship between the parameters erx and $z r x$ allow for coupling by using two data points. The rationale for this may be seen in Equation (4), where the rate of reaction may be expressed as

$$
k(x, T)=k_{c}(T) x^{n}(1-q x)^{m}
$$

where

$$
k_{c}(T)=\exp \left(z r x-\frac{e r x}{R T}\right)
$$

This algorithm performs two steps. First, any parameter (although generally either erx or $z r x$ is chosen) is adjusted for a single temperature $T_{c}$ using the aforementioned single parameter-single point iterative algorithm. Then, the value of $k_{c}$ is determined for this temperature using Eq. (6). Then, the single parameter-single point algorithm is repeated at a different temperature. Preservation of $k_{c}\left(T_{c}\right)$ requires that

$$
e r x=R T_{c}\left(z r x-\ln k_{c}\right)
$$

The resultant adjusted values of erx and $z r x$ provide times to explosion that match two calibration points within the convergence tolerance. Figure 6 depicts what an example simulated curve would look like using this calibration method. 
UCRL-TR-225940

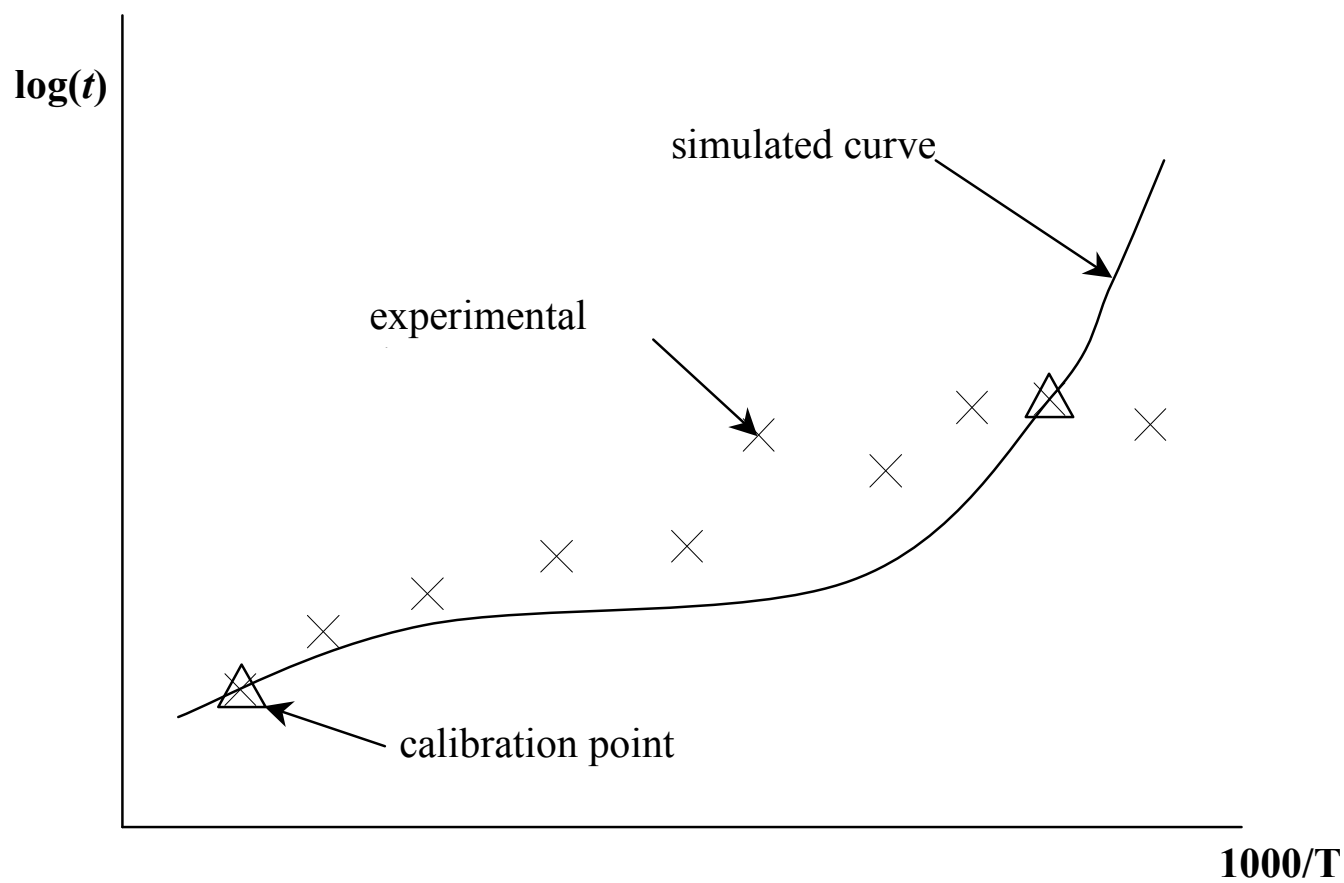

FIGURE 6. Schematic of resultant calibrated curve using coupled erx-zrx two-point calibration.

\section{EZM Three Point Iterative Calibration}

The EZM (erx-zrx-m) three point iterative calibration algorithm is an extension of the coupled erx-zrx two point iterative calibration to allow for additional adjustment of $m$ for a third data point. Figure 7 provides a depiction of how this algorithm works. At each step, the value of $m$ is iterated based on a calculated time for a third data point, where the parameters erx and $z r x$ are previously calibrated for two other points on the curve. The resultant simulated curve passes through three calibration points as shown in Figure 8. Although this technique is powerful, successful completion of the calibrator calls for accurate initial values of erx, $n$, and $q$, and correct bounding limits on $z r x$ and $m$. 
UCRL-TR-225940

Adjust value of $m$ based on single parameter-single point iterative calibration algorithm

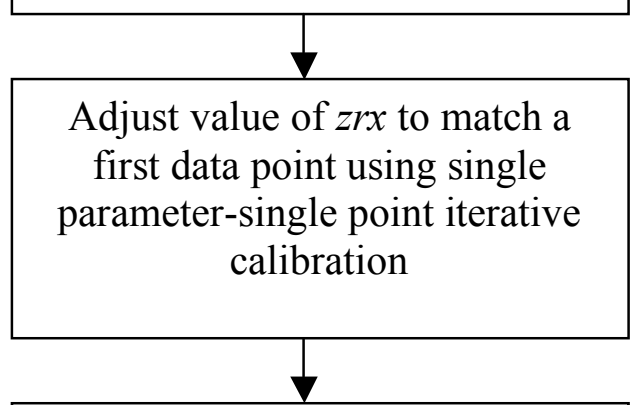

Determine values of erx and $z r x$ based on coupled two-point iterative calibration algorithm for a second temperature.

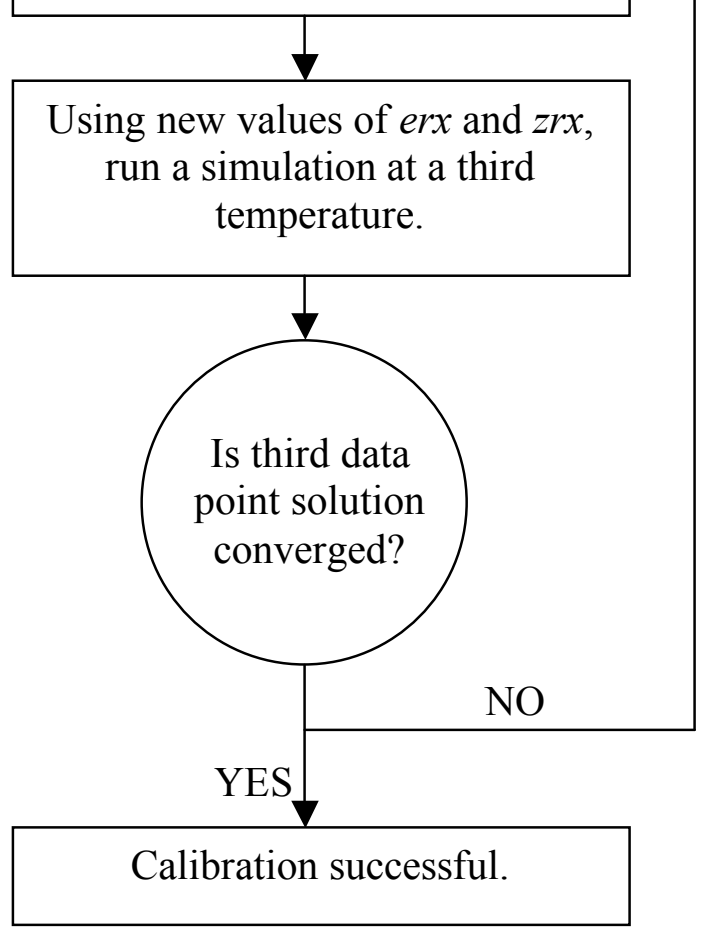

FIGURE 7. EZM iterative calibration algorithm. 
UCRL-TR-225940

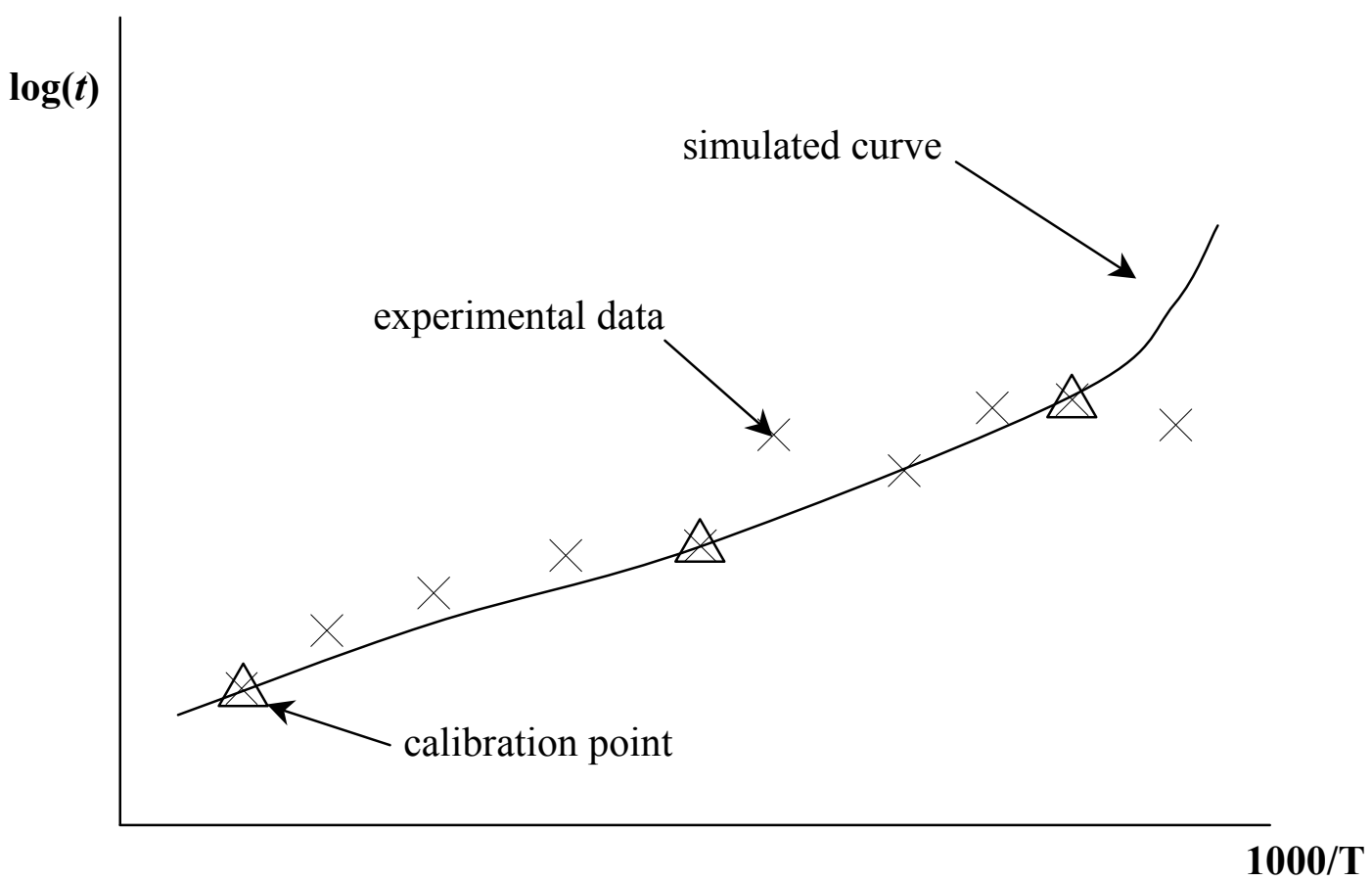

FIGURE 8. Schematic of resultant calibrated curve using EZM calibration.

\section{GLO Calibration}

Although the EZM calibration tool provides an accurate means to calibrate parameters to match experimental data, the algorithm is limited to only three calibration points and to the parameters erx, zrx , and $m$. Use of LLNL's Global Local Optimizer (GLO) code allows for calibration of multiple parameters for multiple calibration points. The GLO code allows for two choices for optimization: global and local [4]. The global optimization tool is generally used for parametric studies, while the local optimization tool searches for local minima using the method of steepest descents. Since kinetic parameter calibration requires a local optimization, the local optimization tool was primarily used in this study. The input to the code is a bracketing window for all parameters (similar to that shown in Figure 2), and initial values for each. Another input is the Figure-of-Merit (FOM) evaluation, which was defined as

$$
F O M=\sum_{i=1}^{N_{p}}\left[\ln \left(t_{\text {cale }} / t_{\text {goal }}\right)\right]_{i}^{2}
$$

where $N_{p}$ data points were used. For each GLO iteration, the parameters were placed in a Perl script, which in turn spawned an ALE3D run for each of the data points, as shown in Figure 9. The results from all ALE3D runs were stored in a text file, which was then read 
UCRL-TR-225940

for post-simulation determination of the FOM. This FOM was then fed back into GLO for consideration of the adjustment of parameters.

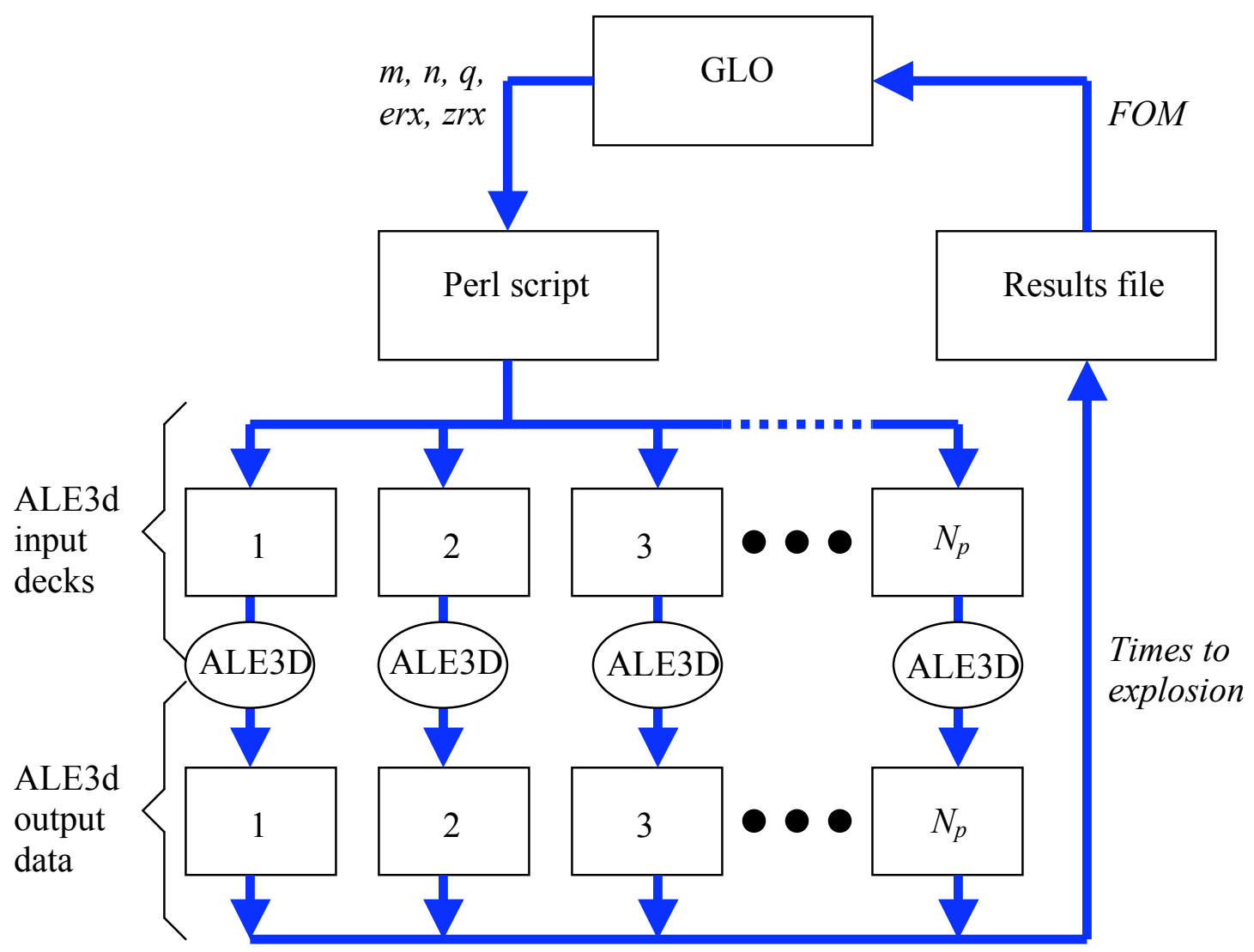

FIGURE 9. Data flow schematic for a GLO run.

The code adjusts the parameters by first determining the gradients along the FOM surface, and then taking a Newton step using the method of steepest descents. If the result of the Newton step yields a FOM larger than at the previous location, then a 1-d minimization subroutine is called to determine the lowest FOM along the 1-d slice between the points before and after the Newton step. Figure 10 shows a typical history of the FOM value for a GLO run where two parameters are calibrated. 
UCRL-TR-225940

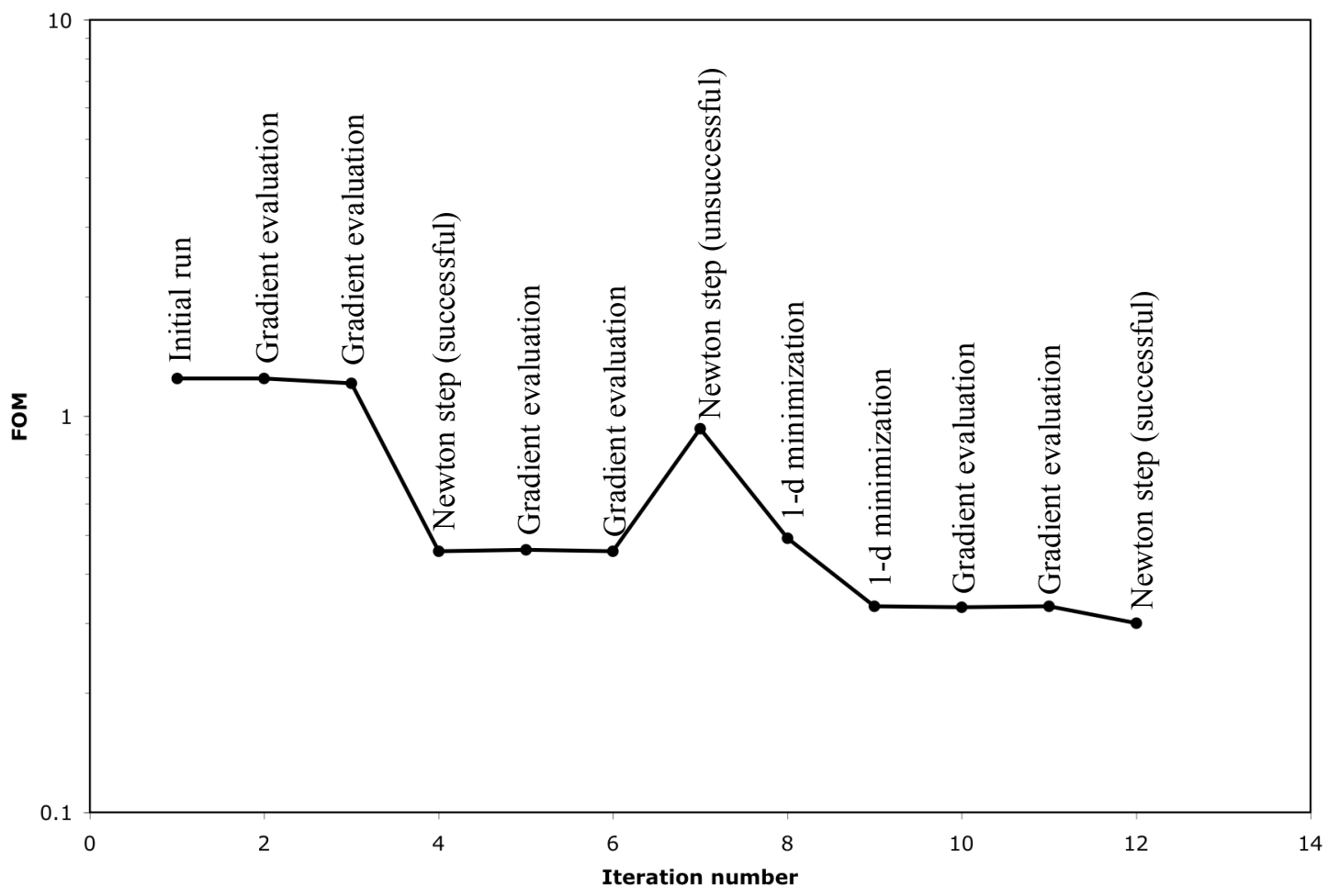

FIGURE 10. Example Figure of Merit adjustment history for a simulation where two parameters are calibrated.

\section{Running the Calibration Codes}

When initializing a calibration process, the first step is to make sure that the working directory contains all the necessary files. These are:

1. PT_inputmake.pl: creates (and has the option to run) the EZM calibrator script.

2. b-ezm_calibrator.pl: base EZM calibrator file (not used directly).

3. auto_optimizer.pl: runs either a one-parameter optimization or a coupled erx-zrx calibration.

4. autovary_odtx.pl: runs an ODTX or STEX simulation at a specified temperature or ramp rate.

5. b-odt $x-1 \times 10-100$. in: base ALE3D input deck (not used directly).

6. b-1x10-kin_bd_v01.in: base ALE3D kinetics input deck (not used directly).

7. b-lx10-mtl_bd_v01. in: base ALE3D material input deck (not used directly).

8. cleaner: list of UNIX commands to remove unnecessary files after a calibration run.

9. sphh.sami: Mesh used in ODTX simulations.

10. glomake.pl: Creates the GLO input file for fine-tuning of kinetic parameters.

11. auto_cleanup.pl: Cleans up directories created during a GLO run. 
Several other files will be created during the calibration process. Figure 11 below sketches out how the codes create new files:

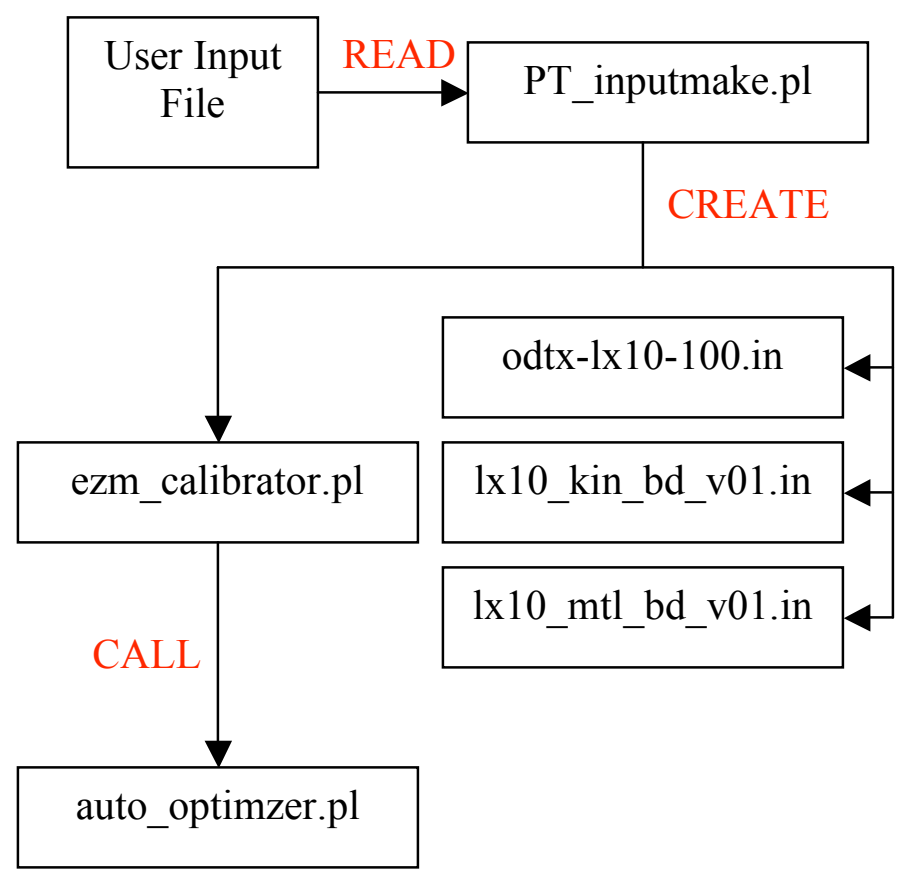

FIGURE 11. Files created during the calibration process.

The second step is to create the appropriate input decks using PT_inputmake.pl. This script reads in the general parameters of the simulation, and creates the appropriate EZM script setup along with the ALE3D input decks. Table 2 lists the parameters to be input to PT_inputmake.pl. The rules for this input deck are as follows:

- Lines with parameter names must include at least one space between the name and value.

- The parameter name must appear prior to the parameter value.

- ODTX data must include at least one space between the name and ODTX temperature, and at least one space between the ODTX temperature and ODTX time.

- Commented lines contain an asterisk "*" in the first column.

- Blank lines are allowed.

- Blank spaces before variable names are allowed but not required.

To run the script, type at the prompt

perl PT_inputmake.pl

and supply the appropriate command-line flags (default values in parentheses, input line flags in brackets):

- Input file name (input.txt)[-i]

- Run the EZM calibrator automatically after creating the ALE3D input deck [-r] 
UCRL-TR-225940

- Obtain help info [-h]

TABLE 2. Input variables to PT_inputmake.p1.

\begin{tabular}{|c|c|c|}
\hline Parameter & Default Value(s) & Description \\
\hline \multicolumn{3}{|c|}{ General Material Properties } \\
\hline matname & gen_mat & Material Name \\
\hline ref_density & 1.0 & Reference density, $\mathrm{g} / \mathrm{cc}$ \\
\hline solid_cv & $1 . e-5$ & $\begin{array}{l}\text { Solid heat capacity at constant volume, } \\
\text { Mbar-cc/(g-K) }\end{array}$ \\
\hline solid_cond & $1 . e-14$ & $\begin{array}{l}\text { Solid thermal conductivity, } \\
\text { Mbar-cc/(cm-us-K) }\end{array}$ \\
\hline prod_cv & $1 . e-5$ & $\begin{array}{l}\text { Products heat capacity at constant volume, } \\
\text { Mbar-cc/(g-K) }\end{array}$ \\
\hline prod_cond & 5.e-15 & $\begin{array}{l}\text { Products thermal conductivity, } \\
\text { Mbar-cc/(cm-us-K) }\end{array}$ \\
\hline prod_energy & 1000 & Energy released by reaction in cal/g \\
\hline prod_coeff & 0.3 & Products gamma-law gas coefficient \\
\hline \multicolumn{3}{|c|}{ Prout-Tompkins Calibration Parameters } \\
\hline pt $\_$p & 9 & Prout-Tompkins parameter $p=-\log _{10}(1-q)$ \\
\hline $\mathrm{pt} \_\mathrm{n}$ & 0 & Prout-Tomkins parameter $n$ \\
\hline itmax_m & 20 & $\begin{array}{l}\text { Maximum number of iterations over the } \\
\text { Prout-Tompkins parameter } m\end{array}$ \\
\hline itmax_z & 20 & $\begin{array}{l}\text { Maximum number of iterations over the } \\
\text { Prout-Tompkins parameter } z r x\end{array}$ \\
\hline tol_m & 0.01 & Convergence tolerance for $m$ \\
\hline tol_z & 0.01 & Convergence tolerance for $z$ \\
\hline pt_mmin & 0 & Minimum value of $m$ \\
\hline pt mmax & 1 & Maximum value of $m$ \\
\hline pt_zmin & 5 & Minimum value of $z r x$ \\
\hline pt_zmax & 30 & Maximum value of $z r x$ \\
\hline ignore_warn & 0 & Ignore warnings from ALE3D $?=1$ for yes \\
\hline verbose & 0 & $\begin{array}{l}\text { Verbose output from the EZM calibrator? } \\
=1 \text { for yes }\end{array}$ \\
\hline convquit & 0 & $\begin{array}{l}\text { Quit EZM calibrator if there's no } \\
\text { convergence? }=1 \text { for yes }\end{array}$ \\
\hline \multicolumn{3}{|c|}{ ALE3D Run Parameters } \\
\hline gen3dexe & /usr/apps/ale3d/bin/gen3d & Path for GEN3D executable \\
\hline ale3dexe & /usr/apps/ale3d/bin/ale3d & Path for ALE3D executable \\
\hline use_srun & 0 & $\begin{array}{l}\text { Use SLURM (srun) to run ALE3D? = } 1 \\
\text { for yes }\end{array}$ \\
\hline
\end{tabular}


UCRL-TR-225940

TABLE 2. Input variables to PT_inputmake.pl (continued).

\begin{tabular}{|c|c|c|}
\hline Parameter & Default Value(s) & Description \\
\hline \multicolumn{3}{|c|}{ ODTX Data } \\
\hline odtx_pts & 2 & Number of ODTX points ( 2 or 3 ) \\
\hline odtx_1 & $0 \quad 0$ & $\begin{array}{c}\text { Parameter 1: ODTX temperature }\left({ }^{\circ} \mathrm{C}\right) \text { for } \\
\text { point } 1 \\
\text { Parameter 2: ODTX time }(\mathrm{sec}) \text { for point } 1\end{array}$ \\
\hline odtx_2 & $\begin{array}{ll}0 & 0\end{array}$ & $\begin{array}{c}\text { Parameter 1: ODTX temperature }\left({ }^{\circ} \mathrm{C}\right) \text { for } \\
\text { point } 2 \\
\text { Parameter 2: ODTX time }(\mathrm{sec}) \text { for point } 2\end{array}$ \\
\hline odtx_3 & $\begin{array}{ll}0 & 0\end{array}$ & $\begin{array}{l}\text { Parameter 1: ODTX temperature }\left({ }^{\circ} \mathrm{C}\right) \text { for } \\
\text { point } 3 \\
\text { Parameter 2: ODTX time }(\mathrm{sec}) \text { for point } 3\end{array}$ \\
\hline
\end{tabular}

Note that if odtx_pts is equal to 2 , then the following occurs automatically:

- Any input for odtx_3 is ignored.

- The parameter pt_p is set to 9 .

- The parameter itmax_m is set to 1 .

- The parameter tol_m is set to 1 .

- The parameters pt_mmin and pt_mmax are set to 1 .

- The parameter pt_n is set to 0 .

A sample input deck to PT_inputmake.pl is seen below. 
* This is a sample input deck for the PT_inputmake.pl.

* All entries here are default values and should be entered in

* B-division units, except the product energy in cal/g. Some of

* these parameters are the same as the default and thus may be

* ommitted.

matname mymat

ref_density 1.61

solidd_cv $1.3 e-5$

solid_cond $9.5 \mathrm{e}-14$

prod_energy 1108

prod_cv $1.1 \mathrm{e}-5$

prod cond $7.9 \mathrm{e}-15$

prod_coef 0.279

* These are used for determining the calibration procedure. pt p 3

itmax_m 20

itmax_z 26

tol_m 0.2

tol z 0.2

pt_mmin 0

pt_mmax 1

pt_n 8

pt_zmin 1

pt_zmax 50

ignore_warn 1

verbose 1

convquit 0

* These commands are for the path of the ale3d executable \&

* whether to use SLURM (srun)

gen3dexe /usr/apps/ale3d/bin/gen3d

ale3dexe /usr/apps/ale3d/bin/ale3d

use_srun 0

* ODTX values should be listed as a temperature (C) and a time

* (sec). The paramter odtx_pts describes the \# of pts to be fit

* to.

odtx_pts 3

odtx_1 25519.8

odtx_2 16556916

odtx_3 $190 \quad 2828.2$

Two example calibrations are provided later in this report. The code that actually performs the calibration is ezm_calibrator.pl. The first few lines of the code contain the input parameters provided by PT_inputmake.pl. The EZM calibration is initiated by typing

perl ezm_calibrator.pl 
UCRL-TR-225940

or by adding the -r flag to the command line for PT_inputmake.pl.

If any calibration fails, then a more involved approach is needed. Any given calibration run may not be successful for a variety of reasons, including:

- The input ranges of $z r x$ or $m$ are invalid.

- More iteration steps may be needed for convergence (if convquit $=1$ ).

- An ALE3D run may list warnings in chemical or thermal convergence, causing the calibrator to stop (if ignore_warn $=0$ ).

The following section describes how to perform the calibration with more control by using the "built-in" perlscripts.

\section{Performing a Point-by-Point EZM Calibration}

The ezm_calibrator.pl code acts as a manager of the perlscript auto_optimizer.pl. The latter code calibrates the kinetics for each individual point. To run auto_optimizer.pl, type at the command prompt

perl auto_optimizer.pl

and supply the appropriate command-line flags (default values in parentheses, input line flags in brackets):

- Reaction name (pt_solid_to_prod)[-r]

- Name of main ALE3D input deck (odtx-lx10-100.in) [-mainf]

- Name of ALE3D kinetics input deck (lx10_kin_bd_v01.in) [-kinf]

- Parameter to be optimized (zrx)[-v]

- Temperature (205.1) [-t]

- Goal time in seconds (23.4) [-g]

- Maximum number of iterations (10)[-i]

- Minimum parameter value (5)[-low]

- Maximum parameter value (50) [-high]

- Explosion time corresponding to minimum parameter value (unknown) [-lowt]

- Explosion time corresponding to maximum parameter value (unknown) [-hight]

- Convergence tolerance (0.01) [-c]

- Pinned temperature in deg C for coupled erx-zrx calibration [-temp_pin]

- Use simple bracketing instead of linear interpolation [-simple]

- Turn off shifting in linear interpolation [-noshift]

- Maximum ALE3D step size in microseconds $\left(10^{5}\right)$ [-stepsize]

- Apply a time limit to the ALE3D runs (2x the goal time) [-time_limit]

- Ignore warning messages option [-ignore_warn]

- Use srun (SLURM) for Linux operating systems [-use_srun]

- Specify GEN3D path (/usr/apps/ale3d/bin/gen3d) [-gen3dexe]

- Specify ALE3D path (/usr/apps/ale3d/bin/ale3d) [-ale3dexe]

Generally, the only command line flags commonly used for a single-parameter singlepoint calibration are -r, - -v, -t, - -g, -low, -high, and -stepsize. The purpose of the -lowt and -hight flags is to allow the user to "restart" the calibration procedure when the 
optimal value for the parameter lies outside the input range. Note that the -d flag provides the current kinetic parameters drawn from the kinetics file, and the -h flag provides help regarding the various input flags.

Example 2 below will show how to perform this calibration.

Running auto_optimizer.pl generates the following output files in addition to the typical ALE3D output files:

- g-mainfile-i.in, where mainfile is the main ALE3D input file, and $i$ is the iteration number: the modified input file for iteration $i$.

- optimized.res: parameter value and corresponding explosion time for each iteration

- warnings . out: any warning messages from ALE3D that terminates the script (deleted if empty at the end of the run)

\section{Performing a GLO Calibration}

A GLO input generator perlscript glomake.pl has been developed as an aid to the user. This perlscript requires a base ALE3D input deck divided into three files: a main file, a materials file, and a kinetics file. The generator copies and modifies these files into the GLO input deck. The following is needed when running glomake.pl (default values in parentheses, and input line flags in brackets):

- Name of reaction (pt_solid_to_prod) [-r]

- Name of main ALE3D input deck (odtx-lx10-100.in) [-mainf]

- Name of ALE3D kinetics input deck (lx10_kin_bd_v01.in) [-kinf]

- Name of ALE3D materials input deck (1x10_kin_bd_v01.in) [-mtlf]

- Name of temperature data points for calibration (datapoints.txt) [-datf]

- Name of GLO input deck to be created (out.gcf) [-o]

- Maximum number of GLO iterations (50) [-max]

- Number of cpu's to be used in a GLO run (1) [-ncpu]

- Use srun (SLURM) for Linux operating systems [-use_srun]

- Specify GEN3D path (/usr/apps/ale3d/bin/gen3d) [-gen3dexe]

- Specify ALE3D path (/usr/apps/ale3d/bin/ale3d) [-ale3dexe]

Multiple reactions and materials are allowed in the materials and kinetics input decks. All changes in the kinetics input decks are applied to the specified reaction. In the data points input, each point is read in as two columns: temperature and goal time.

Commented lines contain an asterisk $(*)$. The same input rules for the PT_inputmake.pl input file apply for the input to glomake.pl as well. An example valid data points input file datapoints . txt is shown below: 


$\begin{array}{ll}\text { * temp } & \text { goal } \\ 300.0 & 12.1 \\ 285.0 & 24.3 \\ 260.3 & 65.9 \\ 250.1 & 571.6 \\ 240.6 & 2842.0\end{array}$

Upon completion of the deck, GLO may be run interactively using

glo out.gcf

where here out.gcf is the name of the GLO input deck, and glo is aliased to the executable for a given machine (see the GLO user manual [4] for details). The GLO input deck name may be set using the -o flag in glomake.pl. This code is also commonly run in the background via

glo out.gcf $>\&$ out.out $</$ dev/null \&

Similar command-line arguments are used for GLO restarts, and further information is available in the GLO user manual [4].

\section{Running a Set of Sequential Runs with the Same Kinetic Parameters}

In many cases, the calibrated parameters are based on a fraction of the total available ODTX data for a given explosive. Therefore, the user may want to compare predictions from the calibrated model to the remaining unused ODTX data points, or verify the accuracy of the calibrated parameters to used ODTX data points. The script autovary_odtx.pl was created for this purpose. This simple script allows the user to run a series of ALE3D simulations at different temperatures (or ramped boundary conditions). Command line flags for this script are:

- Parameter to be adjusted: (temp) or ramp [-v]

- Problem geometry: (0.5sph), 1sph, 2sph, 0.02cy, 2cy, 0.02cy2D, 2cy2D, SITI [-g]

- Number of runs: (1) [-imax]

- Initial parameter value (1) [-init]

- Sequential change in parameter value (1) [-delta]

- Maximum ALE3D step size in microseconds $\left(10^{5}\right)$ [-stepsize]

- Add delta instead of subtract delta for each subsequent run [-add]

- Keep all ALE3D files after run [-noclean]

- Ignore warning messages after run [-ignorewarn]

- Specify a time limit in seconds [-time_limit]

- Use srun (SLURM) for Linux operating systems [-use_srun]

- Specify GEN3D path (/usr/apps/ale3d/bin/gen3d) [-gen3dexe]

- Specify ALE3D path (/usr/apps/ale3d/bin/ale3d) [-ale3dexe]

- Use a list of temperatures instead of a fixed increment [-list] 
The -list flag overrides any of the fixed-increment flags (-imax, -init, and -delta). The user specifies the name of the file with the temperatures and goal values (exactly the same format as the data points file mentioned for glomake.pl above). For example, if the user types the command:

perl autovary_odtx -init 300 -delta 5 -imax 2

the script will provide 3 ALE3D simulations: at 300 C, 295 C, and 290 C. If the user types the same command, except adds the -list flag:

perl autovary_odtx -init 300 -delta 5 -imax 2 -list datapoints.txt

where datapoints.txt is a text file containing two columns: one for temperature and one for the approximate time for explosion. The results from the run are summarized in the file odt $x-1 \times 10$. res.

\section{Examples}

Two examples are now provided that show how the aforementioned codes are used. The first example shows how to calibrate for a series of ODTX data that fall in a straight line, while the second example shows how to use the codes in detail to perform an EZM calibration.

\section{Example 1: Two-Point Calibration}

In this example calibration, the experimental ODTX data for material ExpOne are shown in Figure 12 below. Since the data appear in a straight line, a standard Excel fit-curve relation to the data provides two calibration points at the two extremes of the temperature range: $\left(300^{\circ} \mathrm{C}, 23.4 \mathrm{~s}\right)$ and $\left(220^{\circ} \mathrm{C}, 5739 \mathrm{~s}\right)$. Assume that the thermal properties are the same as the default values in the input deck.

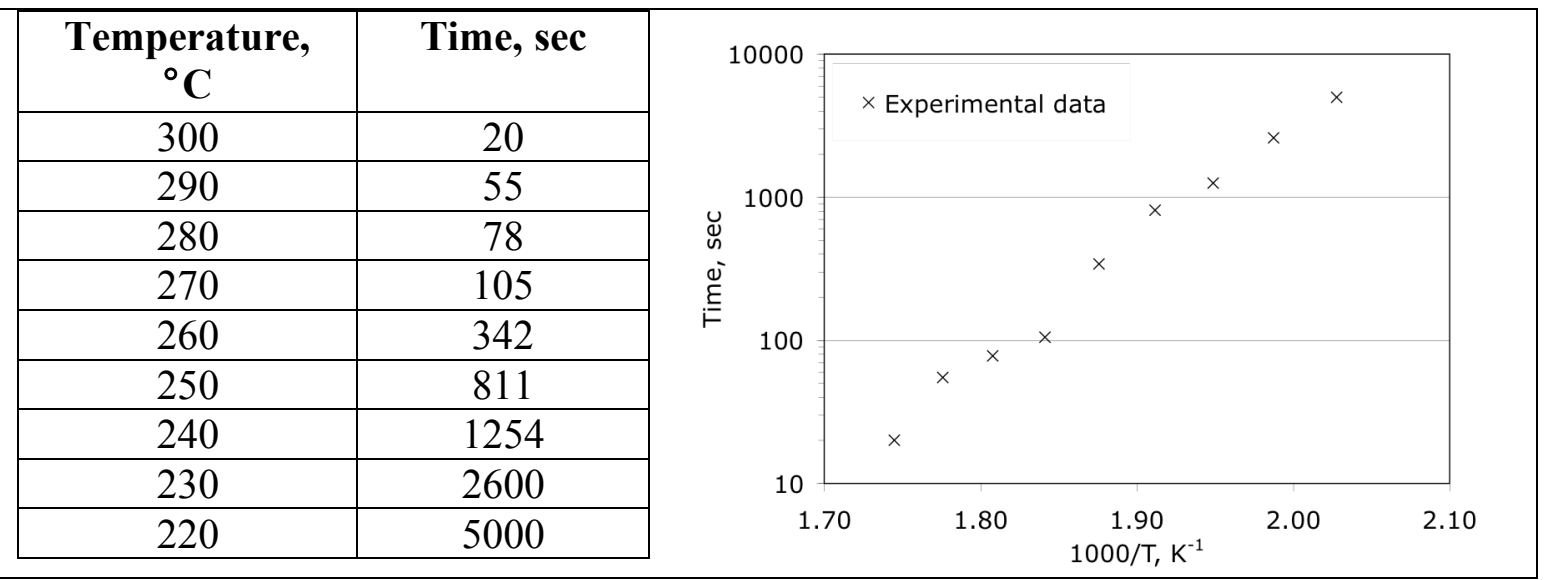

FIGURE 12. Example 1 explosive ODTX data. 
Since the thermal properties are the same as default, then the input deck to PT_inputmake.pl is very simple:

matname Expone

odtx_1 $300 \quad 23.4$

odtx $2 \quad 220 \quad 5739$

Note that this example is applied on the GPS cluster (and hence no srun command is needed) For a Linux cluster, the parameter use_srun should be set to 1 . The input decks for ALE3D and the EZM calibrator is generated using the command

perl PT_inputmake.pl -i input_ex1.txt

where input_ex1.txt is a text file containing the above 3 lines. The EZM calibrator may then be run by typing

perl ezm_calibrator.pl

While the calibrator is running, the code will list a lot of error messages such as "No such file or directory" or "[0] MPI Abort by user Aborting program!" These messages are to be expected and the user should not be alarmed. After a while, the calibrator converges on the first point $(z r x \sim 12.6)$, and then moves on to the second point, where it converges at $(z r x=23.0, e r x=41844)$. It will then re-run at the first point to check for the effect of the new zrx and erx on the first point (the convergence is reduced from 0.01 to 0.09 ). At the end of the run, the EZM calibrator output states the converged parameters (the aforementioned erx and $z r x$, and $m=1)$. These values have been automatically updated in the kinetics file (1x10_kin_bd_v01.in).

After the run, the calibrator has produced a large number of files that can be removed. This is accomplished by using the cleaner by typing

source cleaner

This removes the unwanted ALE3D files. If interested, the user may look at the various * out files that describe the calibrator's attempt to converge for each of the three points.

A check on the calibrated kinetics curve can be produced using

perl autovary_odtx.pl -list data_ex1.txt

where data_ex1.txt is a text file containing the data table similar to that in Figure 12. This creates simulated ODTX times for each of the experimental temperature values.

The resultant plotted simulated data are shown in Figure 13. 


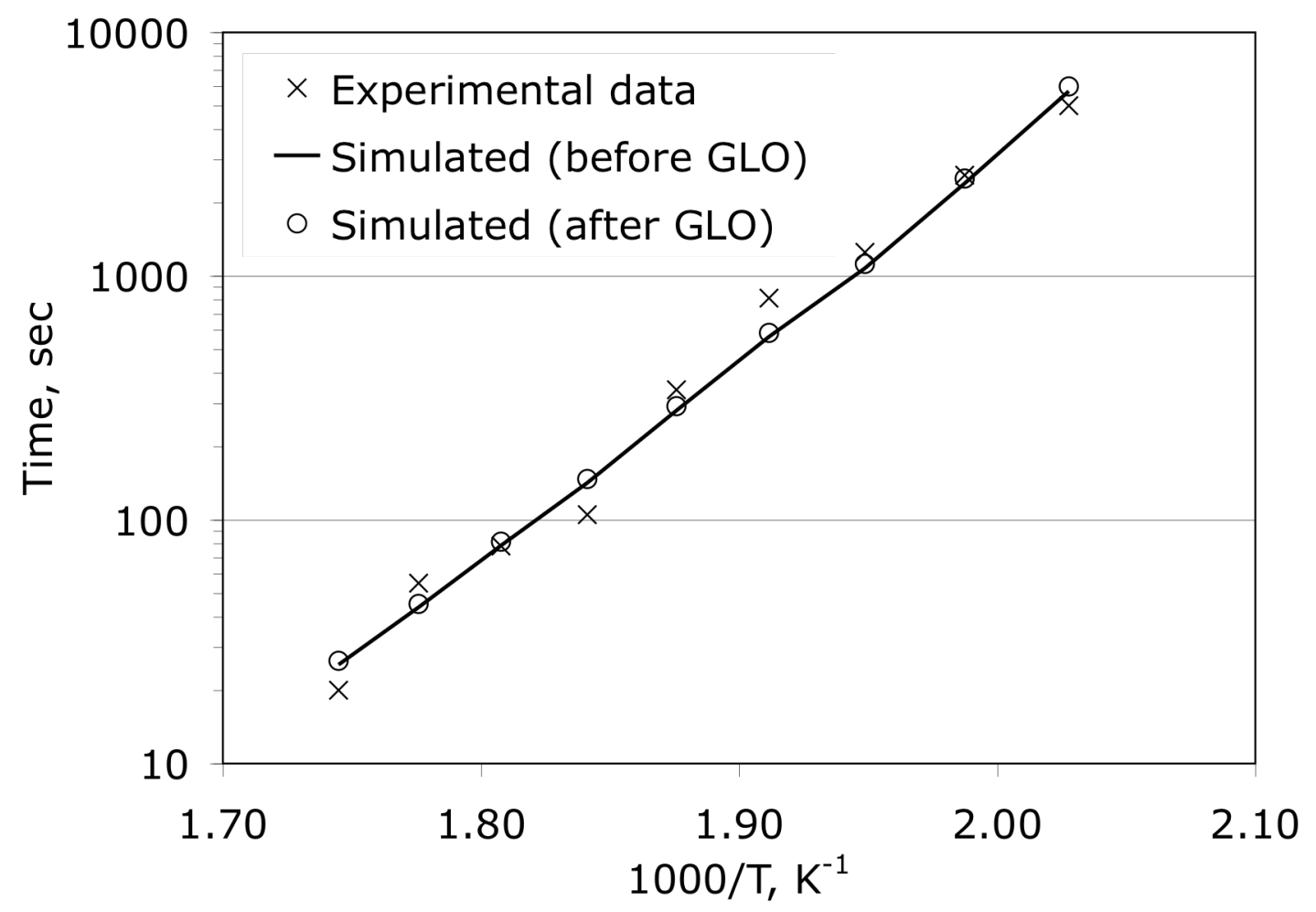

FIGURE 13. Simulated ODTX data using the EZM calibrator compared to experimental data for the example material.

To fine-tune the kinetic parameters using GLO, type at the command prompt

perl glomake.pl -datf data_exl.txt -o exl.gcf

mkdir GLO

cp ex1.gcf GLO

cp sphh.sami GLO

cp auto_cleanup.pl GLO

cd GLO

glo ex1.gcf

Once GLO has finished its run, the excess files in each directory may be removed by typing

perl auto_cleanup.pl

The GLO run has produced several directories beginning with the letter p (e.g. p0001).

To determine which directory to keep, open the ex $1 . u 1$ file and choose the iteration with the lowest figure of merit. The directory contains the values of the kinetic parameters in the varvals file and simulated ODTX times in the results.res file. Figure 12 shows that GLO does not significantly change the kinetic parameters and corresponding ODTX results for this example. 
UCRL-TR-225940

\section{Example 2: Three-Point Calibration}

This second example shows how to calibrate parameters when a bend exists in the data. The data, shown below in Figure 14, show a pronounced dogleg at 1000/T $=1.95$. For this example, three ODTX points are used, and the value of $p$ used is 2 to account for the bend. Assume that the material properties for this explosive, ExpTwo, are the same as the default parameters.

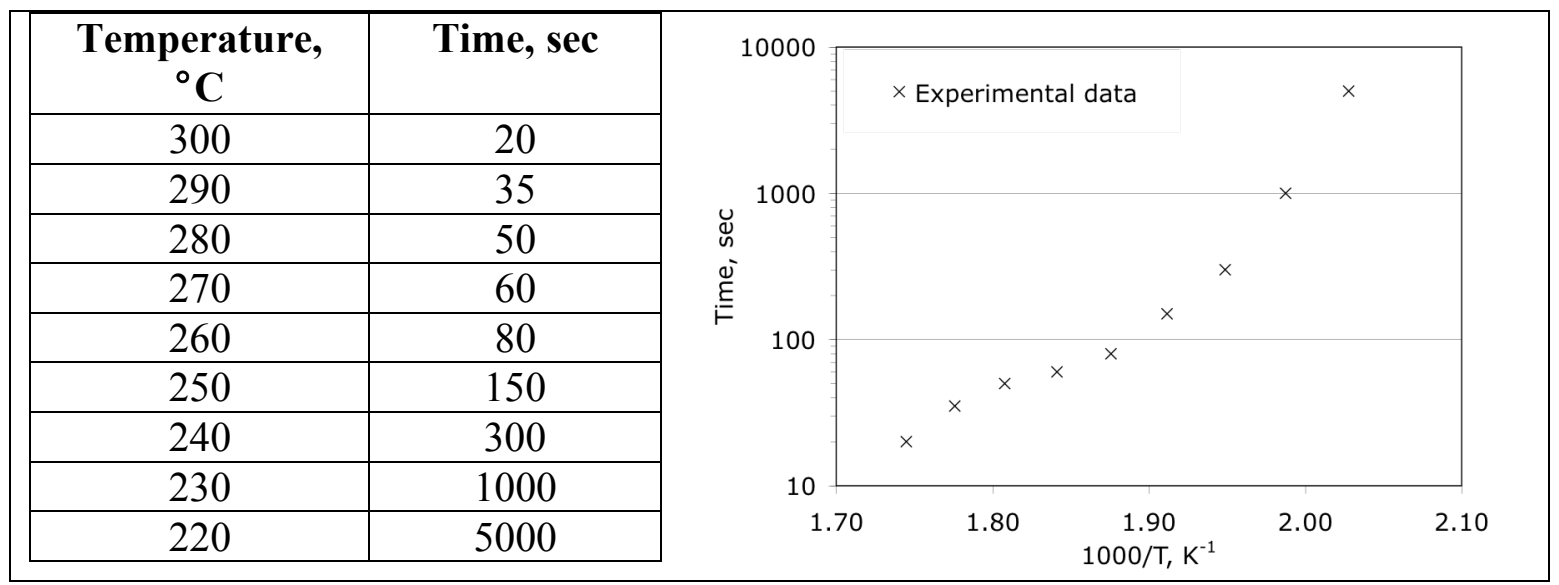

FIGURE 14. Example 2 explosive ODTX data.

First, three representative data points must be chosen. Here, we choose the two extremes of the temperature range and a point at the base of the bend. An example input for this explosive is provided below:

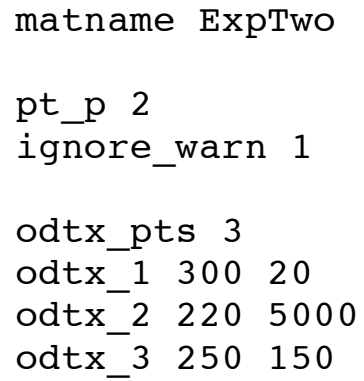

Although running the EZM calibrator will converge to a solution on its own, here we will examine how to manually calibrate using auto_optimizer.pl. The execution of this perlscript for the first point is as follows:

perl auto_optimizer.pl -t 300.0 -g 20 -low 5 -high 30 -stepsize 1. e5

The script opens the kinetics file and determines the reactants and products for the default reaction pt_solid_to_prod. The external boundary temperature is set at $300^{\circ} \mathrm{C}$, the maximum time step size is set to $0.01 \mathrm{sec}$, and the parameter $z r x$ is varied between 5 and 
UCRL-TR-225940

30 until the sample explodes at $20 \mathrm{sec}(+/-1 \%)$. The resultant calibrated value of $z r x$ for the first point is approximately 7.74 .

The coupled erx-zrx calibration may also be implemented via this perlscript. If the above line were used to calibrate $z r x$ for the first point $(300.0 \mathrm{C}, 20 \mathrm{sec})$, then the coupled erx$z r x$ algorithm may be implemented at a second point ( $220 \mathrm{C}, 5000 \mathrm{sec})$, using the temp_pin flag. Update the kinetics file with the value of zrx obtained for the first point, and then enter the following command line to calibrate erx and $z r x$ for the second point using the coupling at the first point:

perl auto_optimizer.pl -t 220 -g 5000 -low 5 -high 30 -stepsize $1 . e 7$-temp_pin 300

This tells the script to attach the pre-calibrated values of erx and $z r x$ to the temperature $300^{\circ} \mathrm{C}$, thus establishing the coupling of the two parameters using Eq. (7), which is then implemented during each successive update to $z r x$. This calibrates erx and $z r x$ to be approximately 42,121 and 18.4 , respectively.

To complete the EZM calibration, update the kinetics file with the new erx-zrx pair, and then test the new parameters with the current value of $m(m=0$ for this iteration) at the third point. Although there are several ways to achieve this, the easiest is by running autovary_odtx.pl at the third point temperature:

perl autovary_odtx.pl -init 250 -stepsize $1 . e 5$-time_limit 300

This results in an explosion time of 126 seconds for $m=0$.

The user then adjusts the value of $m$ in the kinetics file and repeats the above process until convergence is completed for the third point. This process becomes reasonably efficient with use of the history command at the unix prompt to re-run previous auto_optimizer.pl calls. A good general practice is to determine the bounds of $m$, update ezm_calibrator.pl, and then run the latter perl script. Repeating the above process with $m=1$ yields the values $e r x=45,821, z r x=24.9$ and a time to explosion of 206 seconds at $250^{\circ} \mathrm{C}$. Therefore, ezm_calibrator.pl can be updated by changing the lines

\$lowt $=126$;

\$hight $=206$;

Running ezm_calibrator.pl leads to convergence with the following values:
- $\quad e r x=45697$
- $\quad z r x=22.6$
- $m=0.3$ 
Note the additional variables available using ezm_calibrator $\cdot$ pl compared to auto_optimizer.pl. Here, we provided known times for the limits of $m$. In addition, the user can give known calibrated values of $z r x$ at the first point, and known calibrated values of erx and $z r x$ at the second point. This provides a means to save time for restarting a calibration process. Because ezm_calibrator.pl calls auto_optimizer.pl, the latter's output files are created, in addition to the following:

- opt-i-zrx.out, where $i$ is an integer: output from the auto_optimizer call for the first data point for iteration $i$.

- opt-i-zrxerx.out, where $i$ is an integer: output from the auto_optimizer call for the second data point for iteration $i$.

- opt-i-m.out, where $i$ is an integer: output from the auto_optimizer call for the third data point for iteration $i$.

- resezm.res: calculated times for the third data point for each iterated value of $\mathrm{m}$

- orig-kinfile, where kinfile is the kinetics file name: a copy of the original kinetics file (modifications are made to the kinetics file during the calibration process)

Figure 15 below compares the simulated and experimental ODTX values for material ExpTwo. Further refinement of parameters may be done using GLO as described in Example 1.

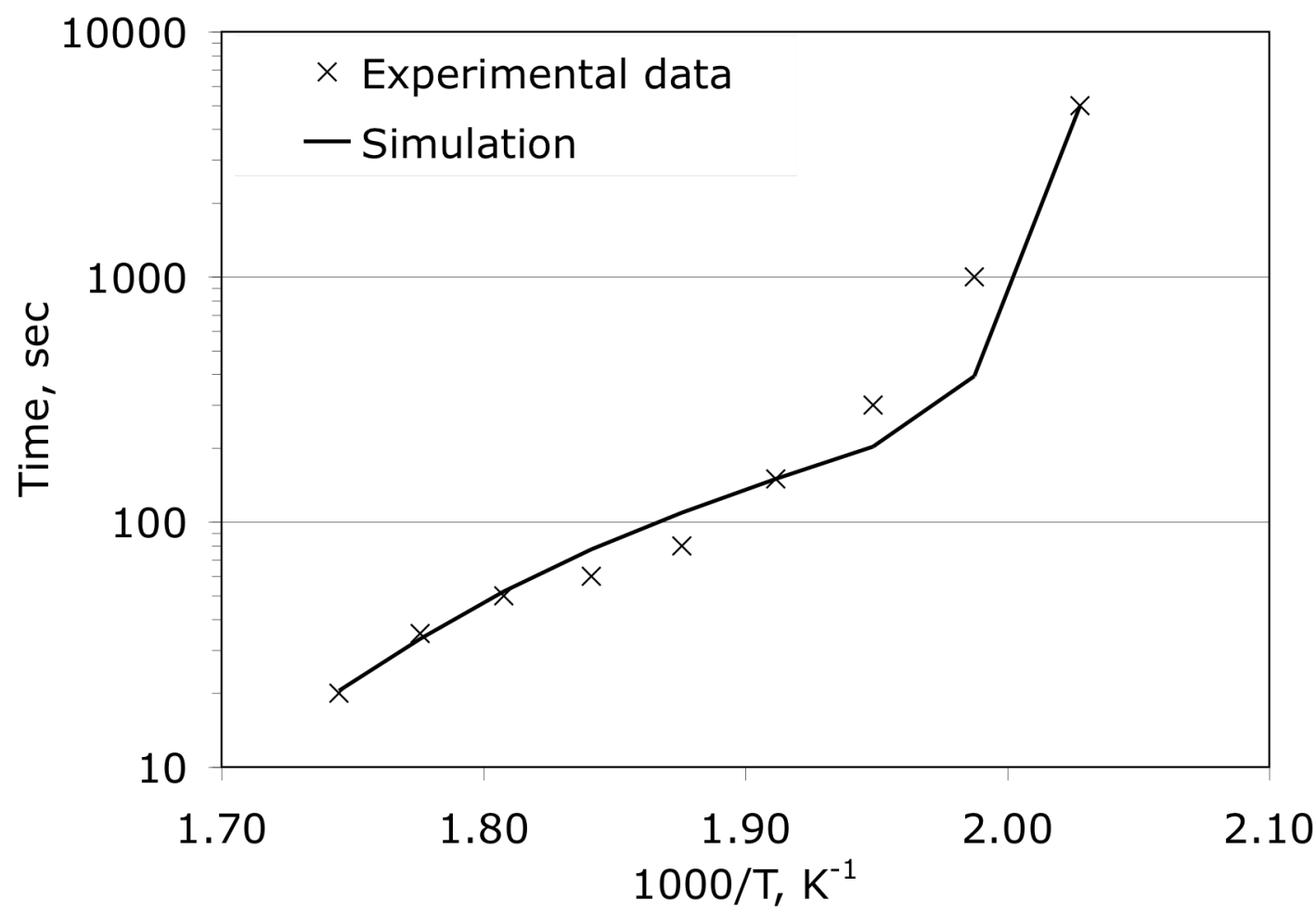

FIGURE 15. Simulated ODTX data using the EZM calibrator compared to experimental data for the material ExpTwo. 
UCRL-TR-225940

\section{References}

1. Wemhoff A. P. and Burnham A. K. (2006) Calibration Methods for ODTX Chemical Kinetics for Various Explosives, Lawrence Livermore National Laboratory, Report UCRL-TR-222032.

2. Burnham A. K. (2000) Application of the Sestak-Berggren Equation to Organic and Inorganic Materials of Practical Interest, J. Therm. Anal. Cal., Vol. 60, pp. 895-908.

3. Owens C., ed. (2005) LLNL Explosives Reference Guide, Lawrence Livermore National Laboratory, Report UCRL-WEB-217165.

4. Murphy M. J. (1999) GLO - Global Local Optimizer User's Manual, Lawrence Livermore National Laboratory, Report UCRL-MA-133858.

5. Wemhoff A. P. and Burnham A. K. (2006) Comparison of the LLNL ALE3D and AKTS Thermal Safety Computer Codes for Calculating Times to Explosion in ODTX and STEX Thermal Cookoff Experiments, Lawrence Livermore National Laboratory, Report UCRL-TR-220687. 